\title{
Effects of cysteamine compound on milk production and hormonal responses of lactating cow during heat stress*
}

\author{
Z. Shen ${ }^{1}$ and R. Zhang \\ Laboratory of Animal Physiology and Biochemistry, Nanjing Agricultural University \\ Nanjing 210095, China
}

\begin{abstract}
In heat-stressed cow received cysteamine compound (Lactonin) the rectal temperature decreased $(\mathrm{P}<0.05)$, milk yield, fat-corrected milk, milk fat and feed conversion rate $(\mathrm{FCR})$ increased $(\mathrm{P}<0.05)$. These changes were companied with trendy $(0.05<\mathrm{P}<0.15)$ of higher milk protein and lower somatic cell count. Additionally, plasma insulin enhanced $(\mathrm{P}<0.01)$ significantly in Lactonin herd $(\mathrm{n}=49)$, whereas $\mathrm{T}_{3}$ and $\mathrm{T}_{4}$ tended $(0.05<\mathrm{P}<0.15)$ to decline. The data show that Lactonin helps heat-stressed cow to maintain a more normal metabolism in hot summer, this positive effect of Lactonin on cow performance was associated with Lactonin-dependent alteration of plasma insulin, $\mathrm{T}_{3}$ and $\mathrm{T}_{4}$.
\end{abstract}

KEY WORDS: cysteamine compound, heat stress, milk production, thyroid hormone, insulin, cow

\section{INTRODUCTION}

In hot summer lactating cow reduced milk yield and feed intake and caused hormones changes in an attempt to attain homeorthermy. Estimated milk yield reduction was $0.2 \mathrm{~kg}$ per unit increase in temperature-humidity index (THI, Ravagnolo and Misztal, 2000), and milk yield and TDN intake declined by 1.8 and $1.4 \mathrm{~kg}$ for each $0.55^{\circ} \mathrm{C}$ increase in rectal temperature (Johnson et al., 1963). Bovine somatotropin (bST) improved milk yield and 3.5\% FCM production in cow during the summer, and changed plasma hormone status (Igono et al., 1992). However, the application of bST to heat-stressed cow is limited due to consumers demand for feed safety. Lactonin is a compound of cysteamine (CS), which is

\footnotetext{
* Supported by China National Ministry of Science and Technology, 2002BA514A-10-2, and Agricultural Resource Bureau of Jiangsu Provine and Shanghai Walcom Bio-Chem Co., Ltd.

${ }^{1}$ Corresponding author: e-mail: zmshen@njau.edu.cn
} 
a component of coenzyme A. in vivo CS is oxidized to taurine. Therefore it is an endogenous substance. CS neutralizes somatostatine, consequently increases levels of growth hormone and blood glucose. The purpose of this study was to investigate the effects of cysteamine compound (Lactonin) on milk production of heat-stressed dairy cow in hot summer.

\section{MATERIAL AND METHODS}

96 Holstein cows, with average lactating day of $210 \mathrm{~d}$, were assigned to 4 groups (G1, G2, G3 and G4) on the basis of their daily milk yield (M) prior to the experiment: $\mathrm{G} 1<24,24<\mathrm{G} 2<28,28<\mathrm{G} 3<32$ and $\mathrm{G} 4>32 \mathrm{~kg} / \mathrm{d}$. In each group the cows were divided randomly into two subgroups: 1. Lactonin treatment (LT, $\mathrm{n}=49)$ and, 2. Control $(\mathrm{n}=47)$.

Lactonin was mixed with maize meal and administered to cow of LT at dose of $3000 \mathrm{U} / \mathrm{d}$ head through the experimental period. In Control the equative maize meal was given without supplement of Lactonin. Experiment was carried out in Shanghai Bright Dairy Farm for 50 d (since July 11 through August 29, 2002), during this period the temperature and humidity in the cowshed was measured tree times per day, milk yield and milk composition was measured every second day and 10 days, respectively. Blood was collected through tail vein at d 10 and d 50 of the experiment.

$\mathrm{THI}=0.72\left(\mathrm{~T}_{\mathrm{d}}+\mathrm{T}_{\mathrm{w}}\right)+40.6 \mathrm{THI}$ : temperature-humidity index; $\mathrm{T}_{\mathrm{d}}$ : dry bulb temperature; $\mathrm{T}_{\mathrm{w}}$ wet bulb temperature.

$3.5 \% \mathrm{FCM}=(0.4324 \times$ milk yield $)+(16.218 \times$ milk fat $)$

The data was expressed as mean \pm SEM. Differences were analysed by $F$ test (Software SPSS) with $\mathrm{P}<0.05$ considered as significant.

\section{RESULTS}

Lactonin improved cow performances. In LT of G1 the rectal temperature decreased $(\mathrm{P}<0.05)$, but milk yield, $\mathrm{FCM}$, and milk protein increased by 21.7 , 24.6 and $25.6 \%$, respectively $(\mathrm{P}<0.05)$, feed conversion rate enhanced by $20.5 \%$ $(\mathrm{P}<0.05)$. Somatic cell count tended to decline $(0.05<\mathrm{P}<0.15)$.

Table 1. Effects of Lactonin on rectal temperature and respiratory rate

\begin{tabular}{lcccccc}
\hline & & G1 & G2 & G3 & G4 & Mean \\
\hline RT & LT & $38.77 \pm 0.08^{*}$ & $39.47 \pm 0.17$ & $39.23 \pm 0.15$ & $39.22 \pm 0.14$ & $39.20 \pm 0.08$ \\
& Cont & $39.20 \pm 0.13$ & $39.01 \pm 0.16$ & $39.13 \pm 0.20$ & $38.87 \pm 0.09$ & $39.05 \pm 0.08$ \\
RR & LT & $76.1 \pm 4.0$ & $81.0 \pm 5.8$ & $78.0 \pm 5.5$ & $80.9 \pm 3.7$ & $81.2 \pm 2.4$ \\
& Cont & $72.3 \pm 4.2$ & $69.3 \pm 3.5$ & $76.0 \pm 3.9$ & $80.4 \pm 4.0$ & $74.5 \pm 2.0$ \\
THI & & & & & & 79.2 \\
\hline
\end{tabular}

RT - rectal temperature, RR - respiratory rate; ${ }^{*} \mathrm{P}<0.05$ 
Mean value of milk fat $(\%)$ in LT groups increased $(\mathrm{P}<0.05)$ and milk protein $(\mathrm{kg} / \mathrm{d})$ tended to be higher than those in Control $(0.05<\mathrm{P}<0.15)$. Somatic cell count tended to be lower $(0.05<\mathrm{P}<0.15)$.

Table 2. Effects of Lactonin on milk production of heat-stressed cow

\begin{tabular}{|c|c|c|c|c|c|c|}
\hline & & G1 & G2 & G3 & G4 & Mean \\
\hline \multirow[t]{2}{*}{ Milk yield, kg/d } & LT & $16.8 \pm 0.8^{*}$ & $19.9 \pm 0.6$ & $21.6 \pm 1.1$ & $26.9 \pm 1.0$ & $21.4 \pm 0.7$ \\
\hline & Cont & $13.8 \pm 1.2$ & $20.3 \pm 0.6$ & $21.5 \pm 1.2$ & $27.7 \pm 0.9$ & $20.7 \pm 0.9$ \\
\hline \multirow[t]{2}{*}{$\mathrm{FCM}, \mathrm{kg} / \mathrm{d}$} & $\mathrm{LT}$ & $17.2 \pm 0.9^{*}$ & $20.4 \pm 0.7$ & $22.7 \pm 1.2$ & $28.5 \pm 1.0$ & $22.3 \pm 0.8$ \\
\hline & Cont & $13.8 \pm 1.3$ & $20.7 \pm 0.9$ & $21.6 \pm 1.2$ & $28.9 \pm 0.9$ & $21.1 \pm 0.9$ \\
\hline \multirow[t]{2}{*}{ Milk fat, $\%$} & LT & $3.67 \pm 0.14$ & $3.67 \pm 0.06$ & $3.83 \pm 0.08^{*}$ & $3.88 \pm 0.10$ & $3.76 \pm 0.05^{*}$ \\
\hline & $\mathrm{Co}$ & $3.40 \pm 0$ & $3.59 \pm 0.11$ & $3.52 \pm 0.09$ & $3.76 \pm 0.07$ & $3.56 \pm 0.05$ \\
\hline \multirow[t]{2}{*}{ Milk protein, $\%$} & LT & & & & & $0.09 \#$ \\
\hline & Cont & $3.16 \pm 0.07$ & $3.08 \pm 0.06$ & $3.00 \pm$ & $2.9 \pm 0.06$ & $3.03 \pm 0.04$ \\
\hline \multirow[t]{2}{*}{ Milk protein, $\mathrm{kg} / \mathrm{d}$} & $\mathrm{LT}$ & $0.54 \pm 0.02 *$ & $0.62 \pm 0.01$ & $0.71 \pm 0.04$ & $0.82 \pm 0.03$ & $0.67 \pm 0.02 \#$ \\
\hline & Con & $0.43 \pm 0.04$ & $0.63 \pm 0.03$ & $0.64 \pm 0.03$ & $0.8 \pm 0.02$ & $0.62 \pm 0.02$ \\
\hline \multirow[t]{2}{*}{$\mathrm{SMC}, 103 / \mathrm{ml}$} & LT & $398 \pm 62 \#$ & $486 \pm 118$ & $420 \pm 76$ & $259 \pm 75$ & $395 \pm 44 \#$ \\
\hline & Cont & $659 \pm 122$ & $655 \pm 163$ & $541 \pm 86$ & $270 \pm 87$ & $529 \pm 62$ \\
\hline \multirow[t]{2}{*}{ FCR } & LT & $0.47 \pm 0.02 *$ & $0.56 \pm 0.02$ & $0.61 \pm 0.03$ & $0.76 \pm 0.03$ & $0.60 \pm 0.02$ \\
\hline & Cont & $0.39 \pm 0.04$ & $0.57 \pm 0.02$ & $0.60 \pm 0.03$ & $0.78 \pm 0.02$ & $0.58 \pm 0.05$ \\
\hline
\end{tabular}

$* \mathrm{P}<0.05, \quad * * \mathrm{P}<0.01, \#-0.05<\mathrm{P}<0.15$; SMC - somatic cell; FCR - feed conversion rate

Table 3. Effects of lactonin on plasma hormones

\begin{tabular}{|c|c|c|c|c|}
\hline & & $\mathrm{T}_{3}, \mathrm{ng} / \mathrm{ml}$ & $\mathrm{T}_{4}, \mathrm{ng} / \mathrm{ml}$ & Insulin, $\mu \mathrm{IU} / \mathrm{ml}$ \\
\hline \multirow[t]{2}{*}{ G1 } & LT & $1.50 \pm 0.07^{\#}$ & $57.73 \pm 4.88^{*}$ & $47.30 \pm 4.16^{* *}$ \\
\hline & Cont & $1.64 \pm 0.04$ & $70.39 \pm 2.26$ & $30.32 \pm 4.58$ \\
\hline \multirow[t]{2}{*}{ G2 } & LT & $1.73 \pm 0.04$ & $68.61 \pm 4.28$ & $47.59 \pm 4.83^{* *}$ \\
\hline & Cont & $1.67 \pm 0.10$ & $66.32 \pm 5.26$ & $25.84 \pm 2.09$ \\
\hline \multirow[t]{2}{*}{ G3 } & LT & $1.67 \pm 0.07$ & $76.22 \pm 4.80$ & $44.75 \pm 5.58$ \\
\hline & Cont & $1.78 \pm 0.05$ & $77.16 \pm 5.31$ & $32.08 \pm 7.16$ \\
\hline \multirow[t]{2}{*}{ G4 } & LT & $1.65 \pm 0.07^{\#}$ & $64.15 \pm 3.36$ & $48.66 \pm 4.10^{* *}$ \\
\hline & Cont & $1.80 \pm 0.05$ & $67.89 \pm 4.50$ & $26.10 \pm 2.83$ \\
\hline \multirow[t]{2}{*}{ Mean } & LT & $1.65 \pm 0.03^{\#}$ & $66.80 \pm 2.27$ & $47.18 \pm 2.21^{* *}$ \\
\hline & Cont & $1.72 \pm 0.03$ & $70.44 \pm 2.27$ & $28.26 \pm 2.32$ \\
\hline
\end{tabular}

$* \mathrm{P}<0.05, * * \mathrm{P}<0.01, \#-0.05<\mathrm{P}<0.15$

Lactonin altered plasma hormone status. Insulin concentration enhanced significantly in all LT groups $(\mathrm{P}<0.01)$, except G3. Whereas concentration of $\mathrm{T}_{4}$ was significantly lower in LT of G1, and mean concentration of $\mathrm{T}_{3}$ tended to be lower in LT groups. 


\section{DISCUSSION}

The upper critical temperature for lactating cows is when the THI exceeds 72 . The ambient THI imposed in this trial, exceeded 76 through whole experiment period, was sufficient to cause heat stress (HS) in the cows (Igono et al., 1992). Administration of Lactonin to cows exhibited resulted lower rectal temperature and higher respiratory rate, indicating cows were able to dissipate a possible greater heat production induced by Lactonin. Likely, Lactonin, being similar with bST, enabled cows to maintain normal body temperatures under HS (Johnson, 1991). Lactonin impacted effects on plasma hormone status. On the one hand Lactonin led to a lower concentrations of $\mathrm{T}_{3}$ and $\mathrm{T}_{4}$. This could avoid cow produce more heat in hot environments. On the other hand, Lactonin increased insulin concentration, which helped cow to maintain a more normal metabolism in hot summer and induced the expression of heat-shock protein gene hsp70 (Takeda et al., 2001). Taken together, Lactonin facilitates dairy cow adapted to hot environments and improves cow performance. In this trial the low yielding cows were more responsive to Lactoin than the high yielding cows, which was also observed in bST experiment.

Lactonin increased milk yield, FCM, milk fat, milk protein and feed conversion rate, but reduced somatic cells of heat-stressed cow during hot summer. These positive effects of Lactonin on cow performance were associated with Lactonindependent alteration of plasma insulin, $\mathrm{T}_{3}$ and $\mathrm{T}_{4}$.

\section{REFERENCES}

Ravagnolo O., Misztal I., 2000. Genetic component of heat stress in dairy cattle, parameter estimation. J. Dairy Sci. 83, 2126-2130

Johnson H.D., Ragsdale A.C., Berry I.L., Shanklin M.D., 1963. Temperature-humidity effects including influence of acclimation in feed and water consumption of Holstein cattle. Missouri Agr. Exp. Sta. Res. Bul. 846

Igono M.O., Bjotvedt G., Sanford-Crane H.T., 1992. Environmental profile and critical temperature effects on milk production of Holstein cows in desert climate. Int. J. Biometeorol. 36, 77-87

Johnson H.D., 1991. Effects of somatotropin on milk yield and physiological responses during summer farm and hot laboratory conditions. J. Dairy Sci. 74, 1250-1262

Takeda T., Tsuura Y., Fujita J., Fujimoto S., Mukai E., Kajikawa M., Hamamoto Y., Kume M., Yamamoto Y., Yamaoka Y., Yamada Y., Seino Y., 2001. Heat shock restores insulinsecretion after injury by nitric oxide by maintaining glucokinase activity in rat islets. Biochem. Biophys. Res. Commun. 284, 20-25 